\title{
In-Situ Lorentz Microscopy with Femtosecond Optical Illumination
}

\author{
Jan Gregor Gatzmann ${ }^{1}$, Tim Eggebrecht ${ }^{2}$, Armin Feist ${ }^{1}$, \\ Vladyslav Zbarsky ${ }^{2}$, Markus Münzenberg ${ }^{2}$, Claus Ropers ${ }^{1}$, Sascha Schäfer ${ }^{1}$ \\ ${ }^{1 .}$ IV. Physical Institute, University of Göttingen, 37077 Göttingen, Germany \\ 2. I. Physical Institute, University of Göttingen, 37077 Göttingen, Germany
}

Ultrafast electron microscopy as a laser-pump/ electron-probe technique allows for the investigation of structural and electronic dynamics occurring at sub-picosecond timescales and nanometer length-scales $[1,2]$. However, current implementations necessitate compromises in electron source brightness compared to conventional electron microscopy techniques. Building on similar concepts in ultrafast surface science [3], in-situ transmission electron microscopy with temporally-structured optical sample excitation, employing femtosecond laser pulse trains, offers a complementary electron microscopy approach to access ultrafast processes.

To this end, we implement free-space-coupled femtosecond sample excitation in a Schottky fieldemission electron microscope (Jeol JEM 2100F) and investigate the optical response of magnetic domain structures with Lorentz microscopy. Specifically, we study laser-induced domain rearrangements in polycrystalline iron thin films (10 nm thickness) on silicon nitride membranes which are pumped with single femtosecond laser pulses $(800 \mathrm{~nm}$ wavelength, $40 \mathrm{fs}$ pulse duration, $50 \mu \mathrm{m}$ focal spot size). By inverting the observed image contrast at large defocus, we reconstruct the local in-plane sample magnetization based on a transport-of-intensity approach [4].

Prior to laser-excitation, the iron thin films display the well-known magnetic ripple domain structure (cf. Fig. 1A), as also observed in Kerr microscopy [5]. Upon optical excitation, at laser fluences below a sharp threshold of about $5 \mathrm{~mJ} / \mathrm{cm}^{2}$, single laser pulses induce local magnetic domain wall. At laser fluences above the threshold, a single laser pulse generates a network of magnetic vortex/anti-vortex (V/AV) structures, as depicted in Fig 1B-D. Subsequent laser pulses lead to nearly complete rearrangement of the V/AV network (left and right panel in Fig 1B). While the network is stable without optical excitation and shows no discernible dynamics on timescales of minutes to hours, vortex/ antivortex annihilation can be triggered by illuminating the sample with laser pulses below threshold. After several low fluence optical pulses, the equilibrium ripple domain structure is recovered. The generation of a V/AV-network is remarkable as it presumably is the result of a partially melted, non-equilibrium spin system which is quickly quenched to a metastable state. Possible processes leading to a V/AV network are discussed on the basis of micromagnetic simulations and with respect to ultrafast all-optical pump-probe experiments. The nature and dynamics of the laser-driven magnetic reorganization will be further experimentally investigated with temporally-structured illumination utilizing femtosecond pulse pairs separated by variable time delays.

In conclusion, we report the optically-induced vortex/anti-vortex generation mapped by in-situ Lorentz microscopy and discuss possible pathways for their generation. 
A

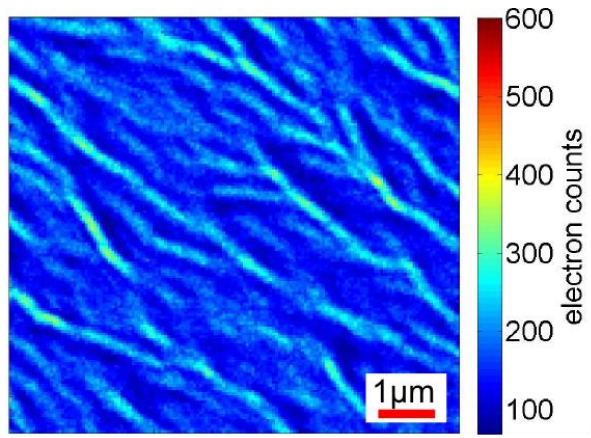

B
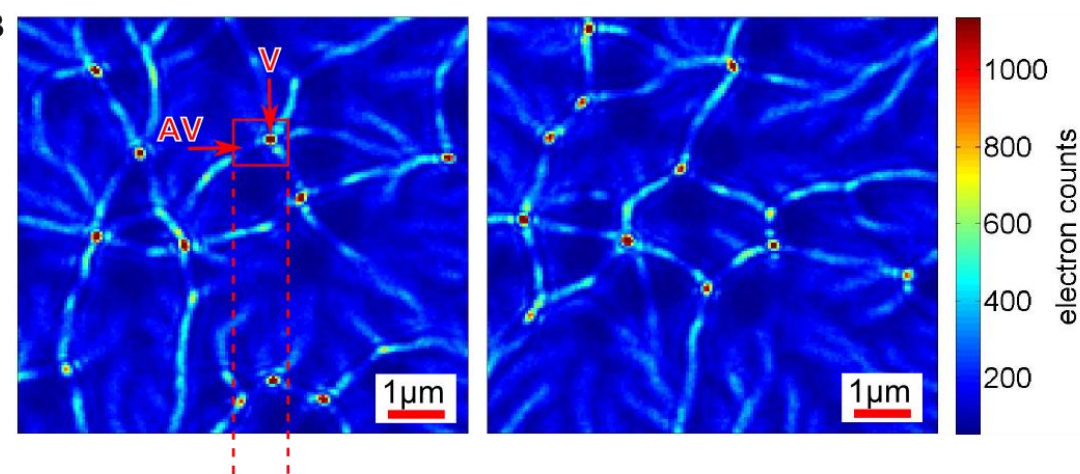
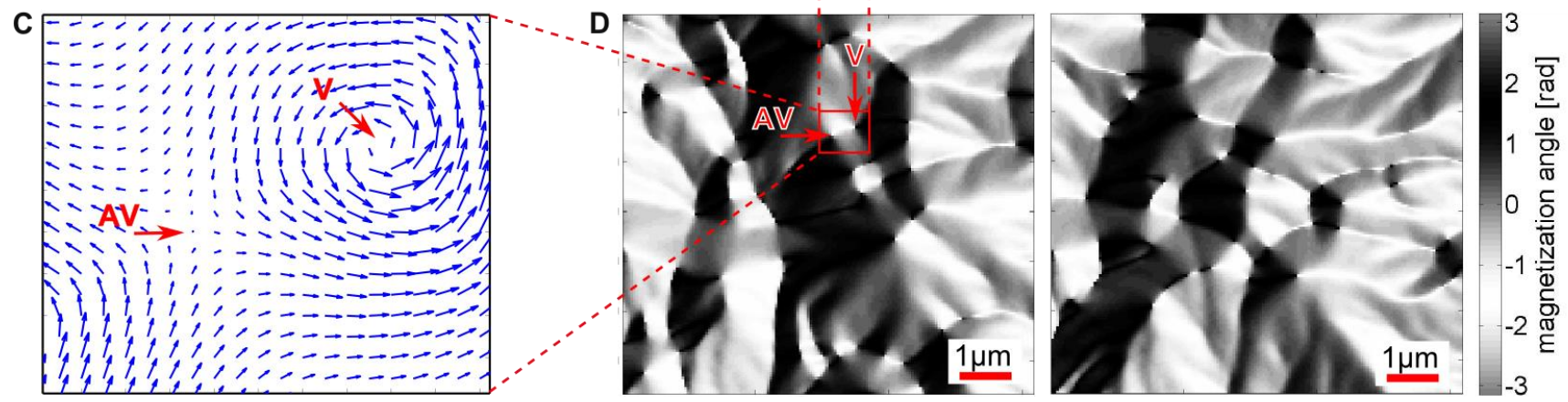

Figure 1: In-situ Lorentz imaging of femtosecond-laser-excited iron thin films. (A) Electron micrograph with Lorenz contrast prior to optical excitation. (B) After single-pulse laser excitation (left panel), a network of vortices and anti-vortices appears. Subsequent laser pulses (right panel) lead to a rearrangement of this network. (C,D) Based on the Lorentz images in (B), the in-plane magnetization is reconstructed. The magnetization around a vortex/anti-vortex pair is depicted in (C) and the corresponding angle of magnetization is shown in (D).

\section{References:}

[1] A. H. Zewail, Science 328 (2010), 187.

[2] H. S. Park , J. S. Baskin and A. H. Zewail, Nano Lett. 10 (2010), 3796.

[3] Bonn et al., Science 285 (1999), 1042.

[4] V. V. Volkov, Y. Zhu and M. De Graef, Micron 33 (2002), 411.

[5] A. Huber and R. Schäfer, "Magnetic Domains: The Analysis of Magnetic Microstructures" (Springer), 452. 